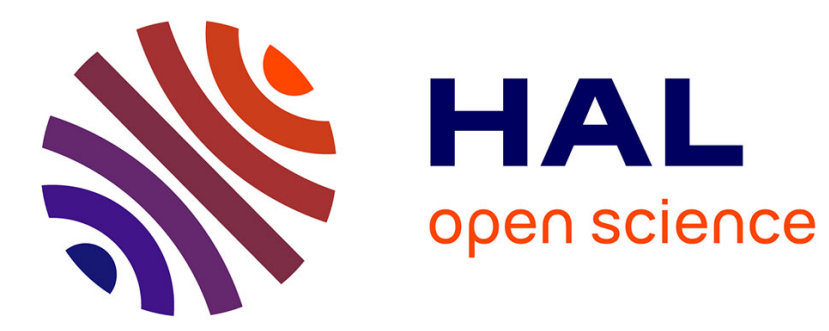

\title{
Virtual Porous Carbons: What are they and what can they be used for
}

Mark James Biggs, Alex V Buts

\section{To cite this version:}

Mark James Biggs, Alex V Buts. Virtual Porous Carbons: What are they and what can they be used for. Molecular Simulation, 2006, 32 (07), pp.579-592. 10.1080/08927020600836242 . hal-00514987

\section{HAL Id: hal-00514987 \\ https://hal.science/hal-00514987}

Submitted on 4 Sep 2010

HAL is a multi-disciplinary open access archive for the deposit and dissemination of scientific research documents, whether they are published or not. The documents may come from teaching and research institutions in France or abroad, or from public or private research centers.
L'archive ouverte pluridisciplinaire HAL, est destinée au dépôt et à la diffusion de documents scientifiques de niveau recherche, publiés ou non, émanant des établissements d'enseignement et de recherche français ou étrangers, des laboratoires publics ou privés. 


\section{Molecular Simulation}

Journal of

Experimental Nanoscience

- Taylor \& Francis

\section{Virtual Porous Carbons: What are they and what can they be used for}

\begin{tabular}{|r|l|}
\hline Journal: & Molecular Simulation/Journal of Experimental Nanoscience \\
\hline Manuscript ID: & GMOS-2005-0089.R1 \\
\hline Journal: & Molecular Simulation \\
\hline Date Submitted by the & 11 -Apr-2006 \\
\hline Complete List of Authors: & $\begin{array}{l}\text { Biggs, Mark; University of Edinburgh, Institute for Materials and } \\
\text { Processes } \\
\text { Buts, Alex; University of Edinburgh, Institute for Materials and } \\
\text { Processes }\end{array}$ \\
\hline Keywords: & $\begin{array}{l}\text { adsorption, anomalous diffusion, elevated freezing, nanoporous } \\
\text { carbons, microporous carbons }\end{array}$ \\
\hline &
\end{tabular}

\section{SCHOLARONE ${ }^{m}$ Manuscripts}


Virtual Porous Carbons: What are they and what can they be used for

\author{
M.J. BIGGS* and A. BUTS \\ Institute for Materials and Processes, University of Edinburgh, King's Buildings, \\ Mayfield Road, Edinburgh, EH9 3JL, Scotland, United Kingdom.
}

We use the term 'virtual porous carbon' (VPC) to describe computer-based molecular models of nanoporous carbons that go beyond the ubiquitous slit pore model and seek to engage with the geometric, topological and chemical heterogeneity that characterises almost every form of nanoporous carbon. A small number of these models have been developed and used since the early 1990s. These models and their use are reviewed. Included are three more detailed examples of the use of our VPC model. The first is concerned with the study of solid-like adsorbate in nanoporous carbons, the second with the absolute assessment of multi-isotherm based methods for determining the fractal dimension, and the final one is concerned with the fundamental study of diffusion in nanoporous carbons.

Keywords: Adsorption; anomalous diffusion; elevated freezing; nanoporous solids; microporous carbons.

Submitted for consideration for Special issue of Molecular Simulation entitled "Atomistic simulation and theory of nanoporous carbons and carbon nanostructures".

\footnotetext{
* Corresponding author: M.Biggs@ed.ac.uk.
} 


\section{Introduction}

We use the term 'virtual porous carbon' (VPC) to describe computer-based molecular models of nanoporous carbons that go beyond the ubiquitous slit pore model and seek to engage with the geometric, topological and chemical heterogeneity that characterises almost every form of nanoporous carbon. We differentiate these from the many other complex models that have been proposed for nanoporous carbons since the early $20^{\text {th }}$ century $[1,2]$ by requiring them to be computer-based and, thus, open to further analysis or use in molecular simulations.

VPC models first started to appear in the early 1990s. Since that time, a number of other models have been advanced and used. Whilst such use has lead to increasing recognition by the community of the various roles VPC models can play, there are some who still argue their complexity makes them of limited use. In light of this and the fact that there is now a reasonable number of reports available in the literature on such models and their use, it is timely to undertake a review and look forward to the future.

A brief overview of molecular models of nanoporous carbons will first be undertaken so as to provide the context for the VPC model developments. The various VPC models will then be briefly reviewed. This will be followed by an overview of their usage to date along with more detailed consideration of three examples drawn from our own work. We conclude with a discussion of future challenges both with regards further development of VPC models as well as their application. 


\section{Background}

As recent reviews $[1,2]$ demonstrate, the history of modelling what we now term nanoporous carbons is a long one starting with the work of Debye and Scherrer [3] in the early $20^{\text {th }}$ century. Many of these models have, of course, never been used directly in the molecular simulation context, but rather as a basis for understanding these solids and phenomena associated with them.

The first molecular simulation studies relating to nanoporous carbons $[4,5]$ were based on the slit pore model, which was itself first proposed by Emmett [6] in 1948 and subsequently confirmed quantitatively as an appropriate model for carbons by others in the mid-1970s [7, 8]. In its most basic form, this model is defined by two parallel semiinfinite blocks of graphite whose separation is equal to the pore width. A nanoporous carbon is typically described in terms of a collection of such pores of varying width whose intersections are unimportant.

The slit pore model has been the workhorse of the field since its introduction and is still used regularly (see, for example, refs. [9, 10] for recent reviews). It is, however, widely recognised that its omission of the many complexities of nanoporous carbons leads to significant errors and limits its usefulness. For example, experimental evidence suggests that the walls of carbon micropores are just a few graphene layers thick [11, 12], which have been shown to yield significantly different adsorption behaviour compared to pores with thick walls $[12,13]$. Experimental evidence also suggests that the pore extents are of the same order as the pore width, leading to significant additional accessible surface area and energetic heterogeneity from graphene edge sites $[14,15]$. These sites additionally play an important role in diffusion processes [16-18], as does 
pore system topology (i.e. pore connectivity, loops and deadend pores) [19]. Pore system topology is also an important source of isotherm hysteresis [20]. Nooks and crannies arising from surface defects can trap molecules [17] and thus be a source of experimentally observed irreversible adsorption [21]. Finally, heteroatoms such as nitrogen, sulphur and oxygen are all likely to cause disruption of pore surfaces [22] and are active sites for polar molecules such as water [23-25].

The slit pore model, despite its inherent shortcomings, is here to stay because of its relative simplicity, comparatively low computational cost, and its indispensable role in the day to day characterisation of carbons where substantially more complex models are unlikely to play a significant part for the foreseeable future. It is for reasons such as these that many workers have endeavoured to address the shortcomings of the model whilst still retaining the same basic framework. Some have recently incorporated pore wall thickness distributions within the context of pore size distribution determination [12], for example. Several workers have included chemical heterogeneity by adding active sites of various types to the pore surfaces (e.g. [23-25]). Others have used nonrectangular cross-sections [26]. Single pore junctions formed by the intersection of slit pores have been used to investigate what effect these may have on adsorption and transport behaviour [17]. A variety of workers have used etched pore surfaces [27, 28]. Finally, Seaton and co-workers [27] have attempted to include the effect of pore system topology by combining the slit pore model with networks.

Whilst these models address to a greater or lesser extent various shortcomings associated with the basic slit pore model, there is still a significant gap between the model and reality. This fact is the motivation for the development of more complex models that seek to bring us closer to reality. By doing this, we gain several capabilities, 
which will be demonstrated with examples in the latter part of this paper. Before doing this, however, we briefly review the various VPC models that have been proposed to date.

\section{Overview of existing VPC models}

Attempts to address the various deficiencies of the slit pore model in a more unified manner - i.e. in a single model framework - were long prevented by the computational challenges arising from the complexity of carbons and the length scales that must be spanned to capture everything from the individual atoms through to the pore system topology. Such challenges were, at least in part, solved by the arrival of the parallel supercomputer in the early 1990s and, more recently, commodity high speed CPUs and large memory chips. There are now several models which go well beyond the ubiquitous slit pore model and seek to engage with the various complexities of real carbons, although the level of engagement inevitably varies across the models and, in all cases, is still far from complete. These models are briefly reviewed here.

The first model that could be reasonably termed a VPC was that of Biggs and Agarwal [29], which was motivated by understanding arising from transmission electron microscopy (TEM) studies (e.g. [30]). These studies suggest that carbons are hierarchical in nature, where polyaromatic molecules combine to form basic structural units (BSUs) that in turn aggregate to form regions of local molecular orientation (LMO) which finally assemble to create the mesoporous structure typical of nanoporous carbons [2]. The dimensions of the BSUs and regions of LMO, the average inter-layer distance within the BSUs and the mis-orientation of the BSUs with the regions of LMO can all be determined experimentally [30-33]. Large uniform BSUs and regions of LMO 
are associated with more ordered, less or even non-porous, carbons. Smaller BSUs and regions of LMO accompanied by greater intra- and inter-LMO disorder are, on the other hand, typical of nanoporous carbons. If the BSUs are sufficiently small in such carbons (i.e. 1-2 layers each of less than 10 rings), then greater curvature will arise from the increased number of 5- and 7-membered rings formed between mismatched BSUs.

The VPC models of Biggs and co-workers are constructed from databases of socalled basic building elements (BBEs). The precise definition of the BBEs has varied over the years. The earliest variant [29] used a relatively small database of 26 different BBEs based on crystallites of five small $(4 \times 5$ rings $)$ graphene layers. The BBEs, which are modelled atomistically, were differentiated by the removal of those carbon atoms that would have overlapped when adjacent to any of the other 25 BBEs. The BBEs were randomly assembled on a cubic lattice to yield solids with a particular porosity. By allowing large volumes to be simulated with relative ease $\left(\right.$ e.g. $\left.100 \times 100 \times 100 \mathrm{~nm}^{3}\right)$, it was possible to capture not only a variety of different pore shapes and surfaces, but also a very wide range of pore sizes and pore system topology effects in a single framework. Improvements in computer power and memory sizes in recent years have removed any real constraints on the nature of the BBEs and, therefore, databases of graphene layers, heterocyclic polyaromatic molecules and functional groups are now used. A variety of methods have been used to assemble these into VPC models. In much of the recent work, which will be discussed in more detail below, an algorithm [34] is used to build fully atomistic models that possess a particular average BSU interlayer spacings, pore wall thicknesses, BSU mis-orientation and porosity.

Another early VPC model was that of Segarra and Glandt [35], which was once again motivated by understanding from TEM studies. The basic building element for 
this model is a circular platelet consisting of a finite number of circular graphene layers with polar edges. These platelets are akin to the BSU. The interaction between the platelets and any interstitial fluid was modelled with a potential function obtained from integration of the solid-fluid pair interaction over the platelet volume and edges (i.e. the atomic detail of the platelets was smeared out). The VPC consisted of an isotropic assembly of non-overlapping randomly orientated and placed platelets obtained by a MC process. The VPC model is defined by four parameters - the platelet radius and thickness, which may be both distributed although this was not done in ref. [35], the overall density of the carbon and the polarity of the platelet edges, all of which can, once again, be measured experimentally.

The late 1990s saw the deployment of a number of algorithms that build models that match, within certain limits, specific atomic-level experimental data. The first such model was that of Foley and co-workers [36], who used the SIGNATURE algorithm [37] to construct through a stochastic process candidate structures with a specific number of carbon and hydrogen atoms by joining together fragments drawn from a library of polyaromatic molecules (they infact describe them as graphene sheets of $m \times n$ hexagons, but both their Figure 6 as well as the existence of hydrogen atoms suggest they are in fact better described as polyaromatic). The candidate structures yielded by the SIGNATURE algorithm often contained unsaturated carbon atoms. Bonds between unsaturated atom pairs from different fragments were, therefore, systematically formed - it is through this process that five and seven membered rings were formed, and hence local curvature in the structure was brought about. Using a classical potential model, the structure was finally relaxed by a local optimiser. 
The SIGNATURE-based approach of Foley and co-workers was not able to produce any model when the $\mathrm{H} / \mathrm{C}$ ratio fell below $\sim 20 \%$, which are typical of many nanoporous carbons. In an attempt to model such carbons, these workers used the PDFFIT algorithm [38] to determine structures that matched the pair distribution function (PDF) obtained from neutron scattering [39]. This algorithm minimises the difference between the PDF of the model and the experimental PDF by a deterministic minimisation least-squares fitting process operating on the unit cell constants, atom positions, atomic site occupancies, and effective thermal factors for a single unit cell of graphite under periodic boundary conditions. Contrary to the SIGNATURE-based approach of Acharya et al. [36], PDFFIT was more successful at modelling carbons prepared at higher temperatures because their PDFs contain significant detail arising from their more ordered structure.

Thomson and Gubbins [40] used a stochastic process to build model carbons that match the experimental PDF of the target carbon. In this method, a solid of required density is built in a fixed volume under periodic boundary conditions. This is done by randomly placing polyaromatic plates of variable shape and distributed size into the volume, roughly aligned in the same direction but with random tilts about their in-plane axes. Each polyaromatic plate is formed from an initial hexagonal ring by adding/deleting hexagonal rings to/from the edge so as to achieve the target solid density and plate size distribution, which is specified by a desired Gaussian mean and standard deviation. Once formed, this initial solid is then subject to a so-called reverse Monte Carlo (r-MC) process in which the polyaromatic plates undergo the following three MC moves until the PDF (or the related structure factor) of the model solid matches its experimental counterpart: (1) translation and re-orientation; (2) ring 
creation/deletion from the plate boundaries, and (3) occasional plate deletion/addition to counteract the effect that ring creation/deletion has on the overall solid density. The attempted MC moves were only accepted if they lead to a reduction in the difference between the model and experimental PDF or structure factor. More recently Gubbins and co-workers [41] have extended the model to randomly incorporate lactone groups at the plate edges so as to enable the fundamental study of systems where polar groups are important (e.g. water adsorption). The approach of Thomson and Gubbins [40] has also been recently used to define the microporous structure of a carbon aerogel model defined by a random assembly of partially overlapping microporous beads [42].

The major problem associated with building models by forcing them to match specific experimental data is their lack of uniqueness - there are potentially many models that will satisfy the experimental data. The classic example of this is the wide spread use of the porosity to build solids where it is obvious that there is an infinite number of ways in which the porosity may be configured, many leading to profoundly different adsorption and transport behaviour. Addressing this lack of uniqueness has characterised much of the most recent work in VPC model development. In principle this can be done by bringing to bear extra discriminating information. The extra information may come in three different guises. The first is to apply constraints that capture various experimental observations or physics. An example of this approach is seen in the latest models from Gubbins and co-workers [43] and in the recent work of Zetterström et al. [44] who both impose constraints that allow the C-C-C bond angles to take on values distributed around $120^{\circ}$ to maintain the strong $\mathrm{sp}^{2}$ character of nanoporous carbons yet allow the formation of five and seven-membered rings which, on the basis of experimental observations, are now thought to exist in carbons. A variety 
of other constraints have also been imposed including the imposition of specific mean coordination numbers on the carbon atoms commensurate with the chemistry of the target solid [43], and exclusion of certain C-C separations [44]. An alternative approach is to use additional experimental data as part of the objective function - this approach has not been practised in its most general from (e.g. fitting spectra from multiple experimental methods) but Zetterström et al. [44] have used Raman spectra data to establish a better starting structure for the reverse MC process compared to the completely random structures used by others. The final choice is to bring to bear some further physics or chemistry - this is done by Peterson et al. [45] who use an environment-dependent potential function in conjunction with the experimental PDFs and static structure factor.

All the approaches reviewed so far are so-called reconstruction methods - they seek to build structures that match experimental characteristics of existing carbons. The alternative is the mimetic approach, where the model is built by mimicking the process used to manufacture the solid. This approach has the advantage that it will, at least in principle, lead to a unique model. The complexity of nanoporous carbons and the processes involved in their production mean, however, that such an approach is very far from trivial. It is, therefore, not surprising that little work has been done in this direction. Relevant quantum [46] and classical [47] molecular simulations of carbonization have both been reported, but none have been concerned with building VPC models for nanoporous carbons. One group has, however, recently published a MC based mimetic approach for the production of nanoporous carbon from polyfurfuryl alcohol (PFA) precursors [48]. 
The reconstruction methods may be broadly divided into two groups. The first group [29, 34, 35] aims to build structures that satisfy meanfield experimental quantities such as porosity, density, average inter-layer spacing and so on. As they build molecular models from super-molecular and mesoscale-level data, this approach can be reasonably termed 'top-down reconstruction'. These top-down reconstruction methods are computationally inexpensive, thus allowing large volumes and mesoscale structure to be modelled rapidly and with relative ease. The second group of reconstruction methods [36, 39, 40, 43-45] build molecular models by engaging directly with the atomic characteristics of the target solid in the form of, for example, the pair distribution function. This direct engagement with the atomic details, or 'bottom-up reconstruction', means the correct detailed atomic structure is likely to be captured provided sufficient information is included. This gain comes at the price of much higher computational expense which limits the volumes that can be reasonably modelled, at least currently.

As we will show in the following section, the models obtained from top-down reconstruction can be used in a variety of very useful ways that inform fundamental understanding and potentially advance practise. They do, however, suffer from the problem that they are unlikely to capture accurately the detailed microstructure of real carbons and, therefore, they will be of limited use in some applications such as, for example, the design of processes that follow the manufacture of the carbons (e.g. catalyst impregnation). In such cases, the bottom-up reconstruction methods will come to the fore, but many improvements in these models are still necessary. For example, the models cannot currently capture mesoscale structure except, possibly, in special cases (e.g. carbon aerogels perhaps). For this to happen, multiscale modelling approaches must be developed, further improvements in the experimental data must come (e.g. high 
resolution and longer-range PDFs), more mesoscale experimental data must be used (e.g. that from SANS and analysis of TEM images), and multiple data must be exploited.

\section{What can VPC models be used for}

\subsection{Overview}

Virtual porous carbon models can be used in four different ways: structural elucidation, fundamental study, assessment of simpler models, and design. Examples of the first three may be found in the literature. A brief review of this literature follows here along with three, more detailed, examples drawn from our own work.

The activity of structural elucidation of carbon structure is as old as the first X-ray experiments on carbons [2]. A large range of models for carbons have been proposed since this time. Whilst some of these are remarkably similar despite their development being independent, many are also fundamentally very different. Computer-aided structural elucidation offers the chance to address this situation by allowing more data to be used when building the models and by speeding the search for possible structures. Whilst arguably all VPC models derived by the bottom-up reconstruction or mimetic approaches may be viewed as attempts at structural elucidation, just a few studies were specifically concerned with this issue. Foley and co-workers were interested in elucidating how the structure of a char derived from polyfurfuryl alcohol (PFA) changed with pyrolysis temperature. They used two approaches. The first, which exploited the H/C ratio (see above) [36], lead to structures whose order increased as the pyrolysis temperature decreased, counter to most experimental evidence including their own (viz. compare Figure 15 in ref. [36] with Figure 2 in ref. [39]). By using PDFs 
rather than the chemistry, Foley and co-workers in their second approach [39] obtained more satisfactory results, indicating that the use of the PDF is preferential, although it was also noted that PDFs are not sufficient for highly disordered carbons. Peterson et al. [45] used additional data and an environment-dependent potential function in their structural elucidation studies to overcome this problem.

Both the groups of Biggs and Gubbins have used VPC models extensively for the fundamental study of adsorption within carbons, and revealed phenomena that cannot be obtained by the slit pore model. For example, Thomson and Gubbins [40] found evidence for capillary condensation in pores of $\sim 14.5 \AA$ which the slit pore model would not predict - Biggs et al. [34] found similar behaviour and proposed the concept of pore space convexity to explain this phenomena. In studying the adsorption of water in a VPC model containing lactone groups, Brennan et al. [41] showed how small amounts of water adsorption on these groups may block pores leading to significant reductions in accessible porosity, a phenomena that is often seen in practise. As will be outlined below in more detail, Biggs et al. [34] found that adsorbate densities of atomic or spherical molecular fluids can significantly exceed those of the bulk liquid and approach those of the bulk random solid despite the disruptive effects of complex pore structures. A similar finding was recently reported by Pikunic et al. [49] using their VPC model.

Whilst it has received less attention, VPC models have also been used in the fundamental study of mass transport in carbon nanopore spaces. The first such studies were those of Biggs and Agarwal [29, 50], who considered the mass transport of atomic and diatomic gases within complex carbon pore spaces using equilibrium molecular dynamics - they showed that transport of gases in carbons is sub-diffusive when the 
porosity approaches the percolation threshold, which is itself a function not only of the solid, but also the fluid and temperature. As we will see below, this work has been more recently extended to higher pressures using equilibrium and non-equilibrium molecular dynamic methods. Pikunic and Gubbins [51] also recently reported on a study of diffusion of fluids as a function of loading in their VPC model using equilibrium molecular dynamics. They observed a maximum in the self-diffusion coefficient with loading and obtained diffusivities over an order of magnitude smaller than those obtained from a slit pore model with the same mean pore size.

Simple models such as the slit pore model still have a real role to play despite the development of the more complex VPC models reviewed here. Perhaps one of the areas where simple models will long be used into the future is characterisation of carbons. This continued use of simple models means it is important to assess them and determine where they are likely to fail. Motivated by this, Biggs and co-workers have used their VPC models to extensively assess both carbon characterisation methods $[52,53]$ and, more recently, pore network models for diffusion [53].

\section{2. $\quad$ More detailed examples}

\subsubsection{Model and simulation details}

We have worked with nearly 40 different VPC models in our recent studies. We restrict attention here to just two of these models, which are shown in Figure 1 along with their cavity size distributions as measured by MC integration with hard spheres. Both models were generated following the procedure given in Biggs et al. [34]. The first, termed $1_{\mathrm{P}}$ (this code is consistent with all other publications), is built using small BSUs of $m=1-3$ parallel evenly spaced graphene domains of size $9.82 \times 12.76 \AA^{2}$ arranged 
randomly in such a way as to achieve a desired porosity and average $d_{002}$. The second model, $1_{\mathrm{P}(15,15)}$ is derived from the first by simply randomly tilting the BSUs up to $\pm 15^{\circ}$ about the $x_{a}$ and $x_{b^{\prime}}$ axes to create a porosity in which no pores are parallel-sided slits and where opposing pore walls can be of differing character (e.g. an armchair surface may in part be opposed by a basal surface). The complex microporosity of both solids effectively decouples the rigid link between pore size and energy that exists in simpler models [34]. Whilst these models have not been built to match the characteristics of any specific carbon, previous work [34] has demonstrated that these and similar models can produce a wide range of isotherm shapes and heat of adsorption loading dependencies that match those observed experimentally.

The fluids were all modelled by a spherical Lennard-Jones (LJ) molecule. Both the fluid-fluid and fluid-solid atom interactions were modelled with a truncated and shifted pair potential [54]

$$
\phi(r)= \begin{cases}\phi_{L J}(r)-\phi_{L J}\left(r_{c}\right) & \text { for } r<r_{c} \\ 0 & \text { for } r \geq r_{c}\end{cases}
$$

with

$$
\phi_{L J}(r)=4 \varepsilon_{i j}\left[\left(\frac{\sigma_{i j}}{r}\right)^{12}-\left(\frac{\sigma_{i j}}{r}\right)^{6}\right]
$$

where $r$ is the distance between the pair of interacting centres, $r_{c}$ is the cut-off radius, and $\varepsilon_{i j}$ and $\sigma_{i j}$ are the $\mathrm{LJ}$ energy and length parameters respectively for interaction between species $i$ and $j$. 
The carbon interaction parameters $\varepsilon_{C}=28 k_{b} \mathrm{~K}$ and $\sigma_{C}=3.4 \AA$ [55] were used. The interaction parameters for the fluids are given below for each example. Fluid-solid interaction parameters were derived using the Lorentz-Berthelot combining rules [54].

Adsorption was simulated by the cavity biased grand canonical Monte Carlo (GCMC) method of Mezei [56]. Points on the adsorption and desorption isotherms are generated in this method by changing the chemical potential, which is related to the bulk phase pressure by standard thermodynamic relations acting on a suitable equation of state for the bulk fluid, details of which also follow below for the various fluids considered. The simulation for each point on the adsorption and desorption isotherms was started using the final state of the previous point, with the first point of the adsorption and desorption isotherms starting from an empty pore structure and the last point of the associated adsorption isotherm respectively. Each point was determined using $50 \times 10^{3}$ equilibration steps per molecule, where a step is one attempted move and one attempted insertion/deletion, followed by at least $\max \left(10^{6}, 50 \times 10^{3}\right.$ steps per molecule, 10 insertions/deletions per molecule) production steps; the first of these applies at low loadings, the second at moderate loadings and the last at high loadings where insertion and deletion are difficult. In the case of suspected phase transitions and other special cases, up to fifty times this number of production steps were used.

Diffusion was simulated using canonical equilibrium molecular dynamics (EMD) [57]. The number of molecules corresponding to the desired bulk pressure were initially inserted using GCMC. Once equilibrated, the molecules were allocated random velocities from a Maxwellian distribution with a mean appropriate to the desired temperature. The net velocity of the ensemble was set to zero and the ensemble temperature re-set to the desired value by direct rescaling of the molecule velocities. 
$50 \times 10^{3}$ timesteps were used for equilibration followed by a further $100 \times 10^{3}$ timesteps for production. The timestep size in all cases was set at $\Delta t^{*}=0.01$, where $t=t^{*} \sigma_{f} \sqrt{m_{f} / \varepsilon_{f}}$ is the reduced time [54].

\subsubsection{Fundamental study of adsorption in carbons}

The use of the slit pore model both before and following the take-up of molecular simulation has greatly improved out understanding of adsorption in porous carbon - the idea that there is an optimal pore 'width' that maximises the storage of a gas on carbon is one good example of improved understanding. The model, however, imposes a symmetry that is unlikely to exist in most (any?) real carbons. This symmetry has several implications, but the one of particular interest here is the structural order that this symmetry places on the adsorbate and the consequent phase behaviour, especially so-called elevated freezing in carbons, where the adsorbed fluid is believed to freeze at temperatures well above that of the bulk freezing point [58]. This freezing phenomena has been well studied using the slit pore model (see Biggs et al. [34] for a recent brief review). Whilst there is some experimental evidence that supports some of the results obtained from the slit pore studies $[58,59]$, there is the question of what happens if the pore symmetry is broken as one would expect in a real carbon - how significant is elevated freezing in this case? We undertook a study of this by carrying out an extensive study of adsorption of $\mathrm{N}_{2}$ at $77 \mathrm{~K}$ on our VPC models. The $\mathrm{N}_{2}$ interaction model parameters $\varepsilon_{N_{2}}=95.2 k_{b} \mathrm{~J}$ and $\sigma_{N_{2}}=3.75 \AA$ [60] were used with the bulk phase equation of state of Smit [61]. An overview of this work and some more recent results are given here whilst the reader is referred to Biggs et al. [34] for more fuller details.

[insert Figure 2 about here] 
The predicted $\mathrm{N}_{2}$ densities at saturation in the solids $\mathrm{P}_{1}$ and $\mathrm{P}_{1(15,15)}$ substantially exceeded that of the bulk liquid state and in fact approached that of a random close packing of spheres. These densities clearly suggest the adsorbate is unlikely to be entirely liquid-like, and may well be solid-like in places, especially if size exclusion effects are accounted for [62]. Further investigation was undertaken by consideration of the singlet distribution function (i.e. the local density distribution) throughout the pore space to reveal solid-like adsorbate did indeed exist in these solids despite the temperature being well above the bulk freezing point, Figure 2. The figure for model $1_{\mathrm{P}(15,15)}$ shows that solid-like adsorbate can even be found in solids where parallel-sided slit pore geometry is virtually non-existent.

[insert Figure 3 about here]

Inspection of the singlet distribution function throughout the filling process shows that solid-like regions exist even well below saturation (e.g. points A on Figure 3). The point $\mathrm{B}$ in this figure shows a region where the adsorbate undergoes a freezing-meltingrefreezing process as the pressure increases. It appears as if this is caused by the need for locally-frozen regions to re-arrange themselves as the space around them fills. These local phase transitions were observed to be reversible with pressure.

\subsubsection{Absolute assessment of characterisation methods}

Characterisation of the porosity of carbons is essential to their design and utilisation. Although adsorption is by far the most widely used means of providing such characterisation, it is not without its problems [63]; these may be broadly described in terms of correctness, consistency (i.e. is the parameter purely related to what it purports to represent or does it 'include' more), and meaningfulness (e.g. what does 'surface 
area' mean in a microporous solid) [52]. Much effort has been directed towards addressing these concerns using relative assessment in which data obtained from two or more methods for a solid are compared (e.g. $[64,65])$. This approach is rarely satisfying for a variety of reasons including, amongst others, the difficulty faced in understanding any observed differences [52].

An alternative to relative assessment is to use a solid whose characteristics are exactly known and for which the interstitial fluid behaviour can be probed in detail. Whilst such an absolute assessment process is (perhaps) experimentally feasible for solids such as zeolites, it is clearly not for ill-defined solids like carbons, which are most in need of assessment. We have, therefore, developed and applied a molecular simulation based methodology for the absolute assessment of adsorption-based characterisation methods, which is illustrated in Figure 4. Briefly, GCMC simulation is used to determine the sorption isotherms for a model fluid in a VPC for which measures of the characteristics are known exactly. The sorption isotherms are then submitted to the method to be assessed and estimates obtained. These estimates are compared with the corresponding exactly known measures and conclusions are drawn regarding the correctness (closed loop in Figure 4) and, if appropriate, meaningfulness and consistency of the methods for the particular model system. Reasons for lack of correctness can be identified and assessment of meaningfulness and consistency can be made by probing the adsorption process at the molecular level; such analysis can be used to suggest improvements to the characterisation method (feedback loop in Figure 4) or an entirely new method that can in turn be assessed.

[insert Figure 4 about here] 
We have applied the absolute assessment methodology illustrated in Figure 4 to a wide range of characterisation methods including the comparison and SPE methods [52], methods based on the Langmuir and BET models [66] and the Polanyi-Dubinin isotherms [67], and methods for determining the pore size distribution [53, 68], connectivity [53, 68], adsorption energies [69] and fractal dimension [53, 70] - an example from the last study is presented here.

The actual fractal dimension and the range of fractility against which the adsorption-based methods are compared are determined using the so-called boxcounting method, which exploits the basic definition of a fractal [71]

$$
N_{b} \propto L^{-D_{b}}
$$

where, in the current context, $N_{b}(L)$ is the number of cubes of size $L$ in a cubic tessellation that intersect the pore surface when it is superimposed on the porous solid, and $D_{b}$ is box-counting dimension, which is in general equal to the fractal dimension. The range of fractility, $w_{l} \leq w_{f} \leq w_{u}$, is defined by the bounds of the linear region of the $\log N_{b}$ vs. $\log (1 / L)$ plot where the slope, which is equal to $D_{b}$, falls between 2 and 3. Application of the box counting approach to model $1_{\mathrm{P}}$ leads to the fractal dimension and range of fractility given at the top of Table 1. The fractal dimension indicates that the surface thoroughly explores 3D space, as expected, whilst the range of fractility corresponds to the size of the nitrogen molecule at the lower end and somewhat below the maximum pore size at the upper end.

[insert Table 1 about here] 
A wide range of different adsorption-based methods have been proposed for the determination of the fractal dimension, $D$. The earliest involves exploiting the extension of equation (3) to adsorption on porous solids [72, 73]

$$
N_{m} \propto \sigma^{-D}
$$

where $N_{m}$ is the monolayer coverage for molecules of size $\sigma$. By determining the monolayer coverage for a range of different sized adsorbates, the fractal dimension and range of fractility can be derived from a plot of $\log N_{m}$ vs. $\log (1 / \sigma)$ in much the same way as in the box counting approach. There are a number of key challenges in applying this multi-isotherm approach to microporous solids. By using spherical molecules with the same LJ energy parameter, one of the main potential reasons for failure of the approach can be removed. The second main challenge is accurate determination of the monolayer coverage, which is thought to be not possible (or even sensible some argue) for microporous materials [63]. To avoid this issue in the first instance, the monolayer coverage was determined directly from the predicted adsorbate structure (see Biggs et al. [52] for details of how this is evaluated). This is, of course, not feasible in the laboratory, but it will provide an indication of the best performance possible from the multi-isotherm approach. The log-log plot of the actual monolayer coverage, $N_{m(a)}$, against the inverse of the molecule size, Figure 5, is linear across most of the molecule size range considered. The best straight line fit to this data (i.e. when the coefficient of determination, $R^{2}$, is maximal) is obtained by omitting the points associated with the two largest molecules, thus indicating that the lower and upper limits of fractility are at least $3 \AA$ and somewhere between 8.25-9 $\AA$ respectively. Inspection of Table 1 shows that the predicted fractal dimension is correct within the degree of uncertainty, 
suggesting that in the best case scenario the multi-isotherm approach will be able to yield a fractal dimension that is accurate to within 5\%, and that it reasonably estimates the fractility bounds.

[insert Figure 5 about here]

Practical application of the multi-isotherm approach requires use of the BET or Langmuir isotherms to determine the monolayer coverages. Whilst this is not recommended for microporous solids [63], it is still widely practised. In such cases, one of the main problems is over what pressure range should the BET or Langmuir isotherms be applied to determine the monolayer coverage. To assess this in the case of the Langmuir isotherm (see [66] for fuller analysis of these isotherms), we considered the: (1) entire pressure range, (2) $0.001 \leq P / P_{0} \leq 0.2$, where the upper limit was always above the knee, and (3) $0.001 \leq P / P_{0} \leq 0.01$. The last of these pressure ranges yielded the most accurate monolayer coverages and fractal dimension, Table 1. The less accurate monolayer predictions obtained from the other two pressure ranges in this instance lead to nonsensical fractal dimensions, Table 1. These results suggest that the correct fractal dimension can be determined by the multi-isotherm approach provided the pressure range for determining the monolayer coverage is correctly identified.

\subsubsection{Fundamental study of diffusion in carbons}

We have recently extended our earlier diffusion work $[29,50]$ to consider the effect of pressure. By way of example, we present here some results obtained for diffusion of methane $\left(\sigma_{C_{4}}=3.7327 \AA\right.$ and $\left.\varepsilon_{C_{4}}=149.92 k_{b} \mathrm{~J}\right)$ [74] in models $1_{\mathrm{P}}$ and $1_{\mathrm{P}(15,15)}$ from $1-40$ bar. 
The components of the mean square displacement (MSD) are shown in Figure 6 for solid $1_{\mathrm{P}}$ at 1 bar (these functions are qualitatively similar for all the other conditions). The monatomic increase of the MSD in each direction indicates that the solid is percolating in all directions. The diffusion rate, which is related to the slope of these lines (see below), clearly differs in the three directions, however, with the rate being substantially less in the $x_{c}$-direction (i.e. the direction normal to the basal plane). This indicates that fluid largely diffuses parallel to the basal planes in the solid (note that this does not mean that pores defined by basal planes are the dominant carriers of the fluid).

[insert Figure 6 about here]

The mean square displacement scales with time as $[75,76]$

$$
R^{2}(t) \propto t^{\alpha}
$$

At $t \rightarrow 0$, the exponent takes a value of $\alpha=2$, corresponding to Newtonian dynamics, whilst as $t \rightarrow \infty$ the exponent takes a value of $\alpha=1$, corresponding to normal (or Fickian) diffusion. The time required to transition between these two regimes is typically very short in the bulk phase, thus making the Fickian diffusion model valid. This need not be the case for diffusion in porous media, however $[29,50,75,76]$. In order to determine how rapidly the systems considered here make this transition, we plot $d\left(\ln R^{2}\right) / d \ln t=\alpha(t)$ against time in Figure 7. This figure clearly shows that the transport behaviour is sub-diffusive (or anomalous) over comparatively long times that increase with pressure. Extrapolation of the data available here suggests the subdiffusive timescale is $\sim 2 \mathrm{~ns}$ at 1 bar, $\sim 4 \mathrm{~ns}$ at 10 bar and an even longer, but 
indeterminate, value at 40 bar. The sub-diffusive timescale is also somewhat longer for model $1_{\mathrm{P}(15,15)}$ compared to its more regular counterpart, model $1_{\mathrm{P}}$.

[insert Figure 7 about here]

The normalised velocity autocorrelation functions (VACFs) in the three directions, which are qualitatively independent of pressure, are shown in Figure $\mathbf{8}$ for both solids at 1 bar. The strong negative tails indicate the molecules do not always move 'forward', but will ultimately double back on themselves. This is caused by tortuous nature of the pore space and the presence of deadend pores. The oscillations in the $x_{c}$-VACF indicates the molecules 'bounce' between the walls of the pores as they move in the other two directions; these frequent collisions mean the velocity in this direction de-correlates rather quickly compared to the other two directions. The oscillations in the $x_{c}$-VACF of the model $1_{\mathrm{P}(15,15)}$ are smoother because the random titling of the plates leads to a distribution of characteristic wall-to-wall distances.

[insert Figure 8 about here]

The diffusion coefficients can be determined from the MSD using the Einstein relationship [31]

$$
D=\lim _{t \rightarrow \infty} \frac{1}{6} \frac{d\left(R^{2}\right)}{d t}
$$

and also from the VACF using the Green-Kubo integral [31]

$$
D=\frac{1}{3} \int_{0}^{\infty} \psi(t) d t
$$

The diffusion coefficients obtained by these two routes were essentially the same except at the highest pressure where there was some difference caused by the fact that 
the MSD and VACF have not been accumulated for a period that exceeds the subdiffusive timescale. The diffusion coefficient estimates for methane at $298 \mathrm{~K}$ in both solids are shown in Figure 9 for the three pressures investigated. The diffusion coefficients are consistent for fluid diffusion in a percolating porous solid. It appears as if the diffusion coefficients do decrease with increasing pressure, although the change is not large. The $20-30 \%$ higher diffusion coefficients of the solid $1_{\mathrm{P}}$ relative to its counterpart arises from the more restricted pore space that comes from tilting of the BSUs.

[insert Figure 9 about here]

\section{Conclusions and the future}

The last decade or more has seen the development of a small number of computer-based nanoporous carbon models that attempt to engage more with the complexities of these solids. There are two broad approaches that may be taken to building these Virtual Porous Carbon (VPC) models - reconstruction where the models are built to match experimentally determined characteristics of the carbons, and mimetic where the model evolves by simulating the actual production process. The complexity of carbon precursors and the production process means little work has been done on the latter. Of the former, there are two groups. The first, which we have term top-down, build models from pre-defined basic structural elements to match super-molecular and mesoscopic experimental data. The second, bottom-up, approach builds models from atomic-level units by matching experimental data such as the pair distribution function of the solids as determined by, for example, X-ray diffraction. 
The bottom-up reconstructive models engage most with the experimental data and are likely to lead to the most realistic and correct structures. However, none of the current models yet engage sufficiently with the experimental data to yield reliable models of nanoporous carbon structure. Engagement with a wider range of experimental data is required in order to make the inverse process more robust. It is also necessary to use experimental data that is capable of informing on the mesoscopic length scales (e.g. SANS; analysis of TEM images) so that the mesoporosity may be captured; this will require a multiscale approach. Whilst a few of the models have included the effect of heteroatoms to some extent, much greater engagement is required with these both in terms of their effect on the structure (e.g. cross-linking) and on phenomena such as adsorption where they can play a significant role.

These complex nanoporous carbon models have been used several times both for fundamental investigation of phenomena associated with carbons, and in assessment of simpler models such as the slit pore. They have also been used for structural elucidation, although there is till significant work required before VPC models can be used in this role satisfactorily. Perhaps the biggest contribution these models can make into the future is in design of value-added nanoporous carbon technologies such as catalysts and electrodes, and the processes that are used in their manufacture. This will become a possibility once sufficiently representative VPC models exist.

\section{Acknowledgements}

We thank the Engineering and Physical Science Research Council of the UK for support of this research (GR/M89539 and GR/R87178). 


\section{References}

[1] P.J.F. Harris. Impact of the discovery of fullerenes on carbon science. Chem. Phys. Carbon, 28, 1 (2002).

[2] T.J. Bandosz, M.J. Biggs, K.E. Gubbins, Y. Hattori, T. Iiyama, K. Kaneko, J. Pikunic, K. Thomson. Molecular models of porous carbons. Chem. Phys. Carbon, 28, 41 (2002).

[3] P. Debye and P. Scherrer, X-ray interference produced by irregularly oriented particles. III. Constitution of graphite and amorphous carbon. Physik Zeitschr., 18, 291 (1917).

[4] W. van Megen and I.K. Snook, Physical adsorption of gases at high pressures III. Adsorption in slit-like pores. Mol. Phys., 54, 741 (1985).

[5] J.P.R.B. Walton and N. Quirke, Modelling the phase behaviour of a fluid within a pore. Chem. Phys. Lett., 129, 382 (1986).

[6] P.H. Emmett, Adsorption and pore-size measurements on charcoals and whetlerites. Chem. Rev., 43, 69 (1948).

[7] F. Stoeckli, The gas-solid interface: Calculations of adsorption potentials in slot-like pores of molecular dimensions. Helv. Chim. Acta, 57, 2195 (1974).

[8] D.H. Everett and J.C. Powl, Adsorption in slit-like and cylindrical micropores in the Henry's law region: A model for microporosity of carbons. J. Chem. Soc. Faraday Trans. I, 72, 619 (1976).

[9] D. Nicholson, Using computer simulation to study the properties of molecules in micropores. J. Chem. Soc. Faraday Trans., 92, 1, 1996.

[10] M.B. Sweatman and N. Quirke, Modelling gas mixture adsorption in active carbons. Mol. Sim., 31, 667, 2005. 
[11] H. Marsh, D. Crawford, T.M. O'Grady and A. Wennerberg, Carbons of high surface area. A study by adsorption and high resolution electron microscopy. Carbon, 20, 419 (1982).

[12] S.K. Bhatia, Density functional theory analysis of the influence of pore wall heterogeneity on adsorption in carbons. Langmuir, 18, 6845 (2002).

[13] X.S. Chen, B. McEnaney, T.J. Mays, J. Alcaniz-Monge, D. Cazorla-Amoros and A. Linares-Solano, Theoretical and experimental studies of methane adsorption on microporous carbons, Carbon, 35, 1251 (1997).

[14] K. Kaneko, C. Ishii, M. Ruike and H. Kuwabara, Origin of superhigh surface area and microcrystalline graphitic structures of activated carbons. Carbon, 30, 1075 (1992).

[15] K. Kaneko, C. Ishii, H. Kanoh, Y. Hanzawa, N. Setoyama and T. Suzuki, Characterization of porous carbons with high resolution $\alpha_{\mathrm{s}}$-analysis and low temperature magnetic susceptibility. Adv. Colloid Interf. Sci., 76-77, 295 (1998).

[16] J.K. Floess and Y. VanLishout, Calculation of adsorption energies in carbon micropores. Carbon, 30, 967 (1992).

[17] C. Lastoskie, K.E. Gubbins and N. Quirke, Pore size distribution analysis and networking: Studies of microporous sorbents. In Characterisation of Porous Solids III, J. Rouquerol, F. Rodríguez-Reinoso, K.S.W. Sing and K.K. Unger (Eds.), pp. 51-60, Elsevier, Amsterdam (1994).

[18] D.M. Ford and E.D. Glandt, Molecular simulation study of the surface barrier effect. Dilute gas limit, J. Phys. Chem., 99, 11543 (1995).

[19] M. Sahimi, G.G. Gavalas and T.T. Tsotsis, Statistical and continuum models of fluid solid reactions in porous-media. Chem. Engng. Sci., 45, 1443 (1990). 
[20] A.V. Neimark, Percolation theory of capillary hysteresis phenomena and its application for characterization of porous solids. In Characterisation of Porous Solids II, F. Rodríguez-Reinoso, J. Rouquerol, K.S.W. Sing and K.K. Unger (Eds.), pp. 67-74, Elsevier, Amsterdam (1991).

[21] S.J. Gregg and K.S.W. Sing, Adsorption, Surface Area and Porosity, p. 197, Academic Press, London (1982).

[22] J. Rodriguez, F. Ruette and J. Laine. Molecular modeling of micropores in activated carbon. Carbon, 32, 1536 (1994).

[23] A.V. Shevade, S. Jiang and K.E. Gubbins, Molecular simulation study of watermethanol mixtures in activated carbon pores. J. Chem. Phys., 113, 6933 (2000).

[24] W. Jin and W. Wang, Computer simulation of adsorption of a Stockmayer molecule chlorodifluoromethane in activated carbon slit pores. J. Chem. Phys., 114, $10163(2001)$.

[25] M. Jorge, C. Schumacher and N.A. Seaton, Simulation study of the effect of the chemical heterogeneity of activated carbon on water adsorption. Langmuir, 18, 9296 (2002).

[26] M.J. Bojan and W.A. Steele, Computer simulation in pores with rectangular crosssections. Carbon, 36, 1417 (1998).

[27] N.A. Seaton, S.P. Friedman, J.M.D. MacElroy and B.J. Murphy, The molecular sieving mechanism in carbon molecular sieves: A molecular dynamics and critical path analysis. Langmuir, 13, 1199 (1997).

[28] A. Vishnyakov, E.M. Piotrovskaya and E.N. Brodskaya, Capillary condensation and melting/freezing transitions for methane in slit coal pores. Adsorption, 4, 207 (1998). 
[29] M. Biggs and P. Agarwal, Mass diffusion of atomic fluids in random micropore spaces using equilibrium molecular dynamics. Phys. Rev. A, 46, 3312 (1992).

[30] A. Oberlin, High resolution TEM studies of carbonization and graphitization. Chem. Phys. Carbon, 22, 1 (1989).

[31] F.G. Emmerich, Evolution with heat treatment of crystallinity in carbons. Carbon, 33, 1709 (1995).

[32] H.S. Shim, R.H. Hurt and N.Y.C. Yang, A methodology for analysis of 002 lattice fringe images and its application to combustion-derived carbons. Carbon, 38, 29 (2000). [33] J.N. Rouzaud and C. Clinard, Quantitative high-resolution transmission electron microscopy: A promising tool for carbon materials. Fuel. Process. Technol., 77-78, 229 (2002).

[34] M.J. Biggs, A. Buts and D. Williamson, Molecular simulation evidence for solidlike adsorbate in complex carbonaceous micropore structures. Langmuir, 20, 5786 (2004).

[35] E.I. Segarra and E.D. Glandt, Model microporous carbons: Microstructure, surface polarity and gas adsorption. Chem. Engng. Sci., 49, 2953 (1994).

[36] M. Acharya, M.S. Trand, J.P. Mathews, S.J.L. Billinge, V. Petkov, S. Subramoney and H.C. Foley, Simulation of nanoporous carbons: A chemically constrained structure. Phil. Mag. B, 79, 1499 (1999).

[37] J.L. Faulon, G.A. Carlson, and P.G. Hatcher, Statistical models for bituminous coal: a three-dimensional evaluation of structural and physical properties based on computer-generated structures. Energy Fuels, 7, 1062 (1993).

[38] T. Proffen and S.J.L. Billinge, PDFIT, a program for full profile structural refinement of the atomic pair distribution function, J. Appl. Cryst., 32, 572 (1999). 
[39] V. Petkov, R.G. DiFrancesco, S.J.L. Billinge, M. Acharya and H.C. Foley, Local structure of nanoporous carbons. Phil. Mag. B, 79, 1519 (1999).

[40] K.T. Thomson and K.E. Gubbins, Modeling structural morphology of microporous carbons by reverse Monte Carlo. Langmuir, 16, 5761 (2000).

[41] J.K. Brennan, K.T. Thomson and K.E. Gubbins, Adsorption of water in activated carbons: Effects of pore blocking and connectivity. Langmuir, 18, 5438 (2002).

[42] S. Gavalda, K.E. Gubbins, Y. Hanzawa, K. Kaneko and K.T. Thomson, Nitrogen adsorption in carbon aerogels: A molecular simulation study. Langmuir, 18, 2141 (2002).

[43] J. Pikunic, C. Clinard, N. Cohaut, K.E. Gubbins, J.M. Guet, R.J.M. Pellenq, I. Rannou and J.N. Rouzaud, Structural modeling of porous carbons: Constrained reverse Monte Carlo method. Langmuir, 19, 8563 (2003).

[44] P. Zetterström, S. Urbonaite, F. Lindberg, R.G. delaplane, J. Leis and G. Svensson, Reverse Monte Carlo studies of nanoporous carbon from TiC. J. Phys.: Condens. Matter, 17, 3509 (2005).

[45] T. Peterson, I. Yarovsky, I. Snook, D.G. McCulloch and G. Opletal, Microstructure of an industrial char by diffraction techniques and reverse Monte Carlo modelling. Carbon, 42, 2457 (2004).

[46] Z. Zhu, G.Q. Lu, J. Finnerty and R.T. Yang, Electronic structure methods applied to gas-carbon reactions, Carbon, 41, 635 (2003).

[47] M.R. Nyden, S.I. Stoliarov, P.R. Westmoreland, Z.X. Guo and C. Jee, Applications of reactive molecular dynamics to the study of the thermal decomposition of polymers and nanoscale structures. Mat. Sci. Eng. A, 365, 114 (2004). 
[48] A. Kumar, R.F. Lobo and N.J. Wagner, Porous amorphous carbon models from periodic Gaussian chains of amorphous polymers. Carbon, 43, 3099 (2005).

[49] J. Pikunic, P. Llewellyn, R. Pellenq and K.E. Gubbins, Argon and nitrogen adsorption in disordered nanoporous carbons: Simulation and experiment. Langmuir, 21, 4431 (2005).

[50] M. Biggs and P. Agarwal, Mass diffusion of diatomic fluids in random micropore spaces using equilibrium molecular dynamics. Phys. Rev. E, 49, 531 (1994).

[51] J. Pikunic and K.E. Gubbins, Molecular dynamics simulations of simple fluids confined in realistic models of nanoporous carbons. Eur. Phys. J. E., 12, 35 (2003).

[52] M.J. Biggs, A. Buts and D. Williamson, Absolute assessment of adsorption-based porous solid characterisation methods: Comparison methods. Langmuir, 20, 7123 (2004).

[53] M.J. Biggs, A. Buts, Q. Cai and N.A. Seaton. Absolute assessment of adsorptionbased microporous solid characterisation methods. In Characterisation of Porous Solids $V I I$, in press.

[54] M.P. Allen and D.J. Tildeseley, Computer Simulations of Liquids, Oxford University Press, New York (1989).

[55] W.A. Steele, The Interaction of Gases with Solid Surfaces, Pergamon, Oxford (1974).

[56] M. Mezei, A cavity-biased $(T, V, \mu)$ Monte-Carlo method for the computer simulation of fluids. Mol. Phys., 40, 901 (1980).

[57] D. Brown and J.H.R. Clarke, A comparison of constant energy, constant temperature and constant pressure ensemble molecular dynamics simulations of atomic liquids, 51, 1243 (1984). 
[58] L.D. Gelb, K.E. Gubbins, R. Radhakrishnan and M. Sliwinska-Bartkowiak, Phase separation in confined systems. Rep. Prog. Phys., 62, 1573 (1999).

[59] H.K. Christenson, Confinement effects on freezing and melting. J. Phys.: Condens Matter, 13, R95 (2001).

[60] J.P.R.B. Walton and N. Quirke, Capillary condensation: A molecular simulation Study. Mol. Sim., 2, 361 (1989).

[61] B. Smit, Phase diagrams of Lennard-Jones fluids. J. Chem. Phys., 96, 8639 (1992).

[62] P.J.M. Carrott, R.A. Roberts and K.S.W. Sing, Molecular packing in slit-shaped and cylindrical pores. Chem. Ind., 24, 855 (1987).

[63] J. Rouquerol, D. Avnir, C.W. Fairbridge, D.H. Everett, J.H. Haynes, N. Pernicone, J.D.F. Ramsay, K.S.W. Sing, K.K. Unger. Recommendations for the characterization of porous solids. Pure \& Appl. Chem., 66, 1739 (1994).

[64] G. Rychlicki, A.P. Terzyk and J.P. Łukaszewicz, Determination of carbon porosity from low-temperature nitrogen adsorption data. A comparison of the most frequently used methods. Colloids Surfaces A, 96, 105 (1995).

[65] M. Kruk, M. Jaroniec and J. Choma, Comparative analysis of simple and advanced sorption methods for assessment of microporosity in activated carbons. Carbon, 36, 1447 (1998).

[66] M.J. Biggs, A. Buts and D. Williamson, Absolute assessment of adsorption-based porous solid characterisation methods: Langmuir and BET isotherms. In preparation. [67] M.J. Biggs, A. Buts and D. Williamson, Absolute assessment of adsorption-based porous solid characterisation methods: Polanyi-Dubinin isotherms. In preparation. 
[68] Q. Cai, A. Buts, N.A. Seaton and M.J. Biggs, An evaluation of the methods for determining the pore size distribution and the network connectivity of nanoporous carbons by characterizing two model carbons. In preparation.

[69] M.J. Biggs, A. Buts and D. Williamson, Absolute assessment of adsorption-based porous solid characterisation methods: Adsorption energetics. In preparation.

[70] M.J. Biggs, A. Buts and D. Williamson, Absolute assessment of adsorption-based porous solid characterisation methods: Fractal dimension. In preparation.

[71] B.B. Mandelbrot, The Fractal Geometry of Nature, Freeman, San Francisco (1982).

[72] P. Pfeifer and D. Avnir, Chemistry in noninteger dimensions between two and three. I. Fractal theory of heterogeneous surfaces. J. Chem. Phys., 79, 3558 (1983).

[73] D. Avnir, D. Farin and P. Pfeifer, Chemistry in noninteger dimensions between two and three. II. Fractal surfaces of adsorbents. J. Chem. Phys., 79, 3566 (1983).

[74] M.V. López-Ramon, J. Jagiełło, T.J. Bandosz and N.A. Seaton, Determination of the pore size distribution and network connectivity in microporous solids by adsorption measurements and Monte Carlo simulation. Langmuir, 13, 4445 (1997).

[75] Y. Gefen, A. Aharony and S. Alexander, Anomalous diffusion on percolating clusters. Phys. Rev. Lett., 50, 77 (1983).

[76] R. Muralidhar, D. Ramkrishna, H. Nakanishi and D. Jacobs, Anomalous diffusion: a dynamic perspective. Physica A, 167, 539 (1990). 


\section{Tables}

\begin{tabular}{lcccc}
\hline \multirow{2}{*}{ Method } & \multicolumn{5}{c}{ Parameter } \\
\cline { 2 - 5 } & $D$ & $w_{l}(\AA)$ & $w_{u}(\AA)$ & $R^{2}$ \\
\hline $\mathrm{BC}^{1}$ & $2.8445 \pm 0.0095$ & 3.84 & 11.16 & 0.9999 \\
$\mathrm{MI}^{2 \mathrm{a}}$ & $2.8704 \pm 0.1275$ & 3.00 & $8.25-9.00$ & 0.9845 \\
$\mathrm{MI}^{2 \mathrm{~b}}$ & $3.2396 \pm 0.0841$ & 3.00 & $8.25-9.00$ & 0.9947 \\
$\mathrm{MI}^{2 \mathrm{c}}$ & $3.2738 \pm 0.0918$ & $3.00-3.375$ & $8.25-9.00$ & 0.9945 \\
$\mathrm{MI}^{2 \mathrm{~d}}$ & $2.8994 \pm 0.0863$ & 3.00 & $8.25-9.00$ & 0.9930 \\
\hline
\end{tabular}

Table 1. Fractal dimensions, $D$, lower and upper bounds on the ranges of fractility, $w_{l}$ and $w_{u}$ respectively, and the coefficient of determination, $R^{2}$, obtained for the model $1_{\mathrm{P}}$ using various methods. Notes: (1) Estimates from box-counting (BC) method; all other results should be compared against these. (2) Estimates from multi-isotherm (MI) method using a number of different monolayer coverages derived from isotherms for a range of spherical LJ molecules where $\varepsilon=95.2 k_{b} \mathrm{~J}$ and $3.00 \leq \sigma \leq 9.75 \AA$ : (a) actual monolayer coverage; (b) monolayer coverage obtained from fitting Langmuir isotherm to $P / P_{0} \leq 0.95$; (c) monolayer coverages obtained from fitting Langmuir isotherm to $0.001 \leq P / P_{0} \leq 0.2$; (d) monolayer coverages obtained from fitting Langmuir isotherm to $0.001 \leq P / P_{0} \leq 0.01$. 


\section{Figure captions}

Figure 1. Top and side views, isoenergy surface (with corner cut-away to reveal nature of porosity inside) and cavity size distribution for models $1_{\mathrm{P}}$ (upper) and $1_{\mathrm{P}(15,15)}$ (lower).

Figure 2. Three $x_{a}-x_{c}$ planes $\sim 1.875 \AA$ (i.e. $\sim 0.5 \sigma_{f}$ ) apart through the singlet distribution function $(\mathrm{SDF})$ for model $1_{\mathrm{P}}$ (left) and model $1_{\mathrm{P}(15,15)}$ (right) at saturation. A log colour scale has been used to facilitate presentation of solid-like and fluid densities in a single plot. The solid atoms within $\pm 1.875 \AA$ of the plane are shown in red and the inaccessible regions of each plane are shaded to demonstrate that all the accessible volume is occupied (note that many of the unfilled spaces in one plane are matched by highlylocalised adsorbate in adjacent planes). The circled regions indicate liquid-like (A), well-localised high density (B) and solid-like (C) adsorbate (after Biggs et al. [34]).

Figure 3. A plane $\left(x_{c}=74.06 \AA\right)$ through the singlet distribution function (SDF) of model $1_{\mathrm{P}}$ for a number of loadings: (a) $P / P_{0}=2.0 \times 10^{-5}$; (b) $P / P_{0}=1.4 \times 10^{-4}$; (c) $P / P_{0}=9.0 \times 10^{-4}$; (d) $P / P_{0}=5.9 \times 10^{-3}$; (e) $P / P_{0}=4.0 \times 10^{-2}$; (f) $P / P_{0}=1.0 \times 10^{-1}$. A log colour scale has been used to facilitate presentation of solid-like and fluid densities in a single plot. The solid atoms within $\pm 1.875 \AA$ of the plane are shown in red. See text for discussion pertaining to the circled regions.

Figure 4. Absolute assessment methodology (after Biggs et al. [52]).

Figure 5. Actual monolayer coverage at $77 \mathrm{~K}$ for model $1_{\mathrm{P}}$ as a function of adsorbate molecule size (the LJ energy parameter is equal to that of $\mathrm{N}_{2}, \varepsilon_{N_{2}}=95.2 k_{b} \mathrm{~J}$, in all 
cases). The line of best fit with the maximal coefficient of correlation is shown along with the resultant range of fractility.

Figure 6. The three components of the reduced mean square displacement (MSD) for methane in model $1_{\mathrm{P}}$ at 1 bar and $298 \mathrm{~K}$.

Figure 7. Variation of the exponent $\alpha$ (see equation (5)) with time for methane at $298 \mathrm{~K}$ in models $1_{\mathrm{P}}$ (left) and $1_{\mathrm{P}(15,15)}$ (right) at the three pressures considered.

Figure 8. The $x_{a}$ (solid line) $x_{b}{ }^{\prime}$ (dotted line) and $x_{c}$ components of the normalised velocity autocorrelation function (VACF) for methane at $298 \mathrm{~K}$ and 1 bar in models $1_{\mathrm{P}}$ (left) and $1_{\mathrm{P}(15,15)}$ (right).

Figure 9. Variation of diffusion coefficient with pressure of methane at $298 \mathrm{~K}$ in models $1_{\mathrm{P}}\left(\right.$ closed circle) and $1_{\mathrm{P}(15,15)}$ (open circle). 

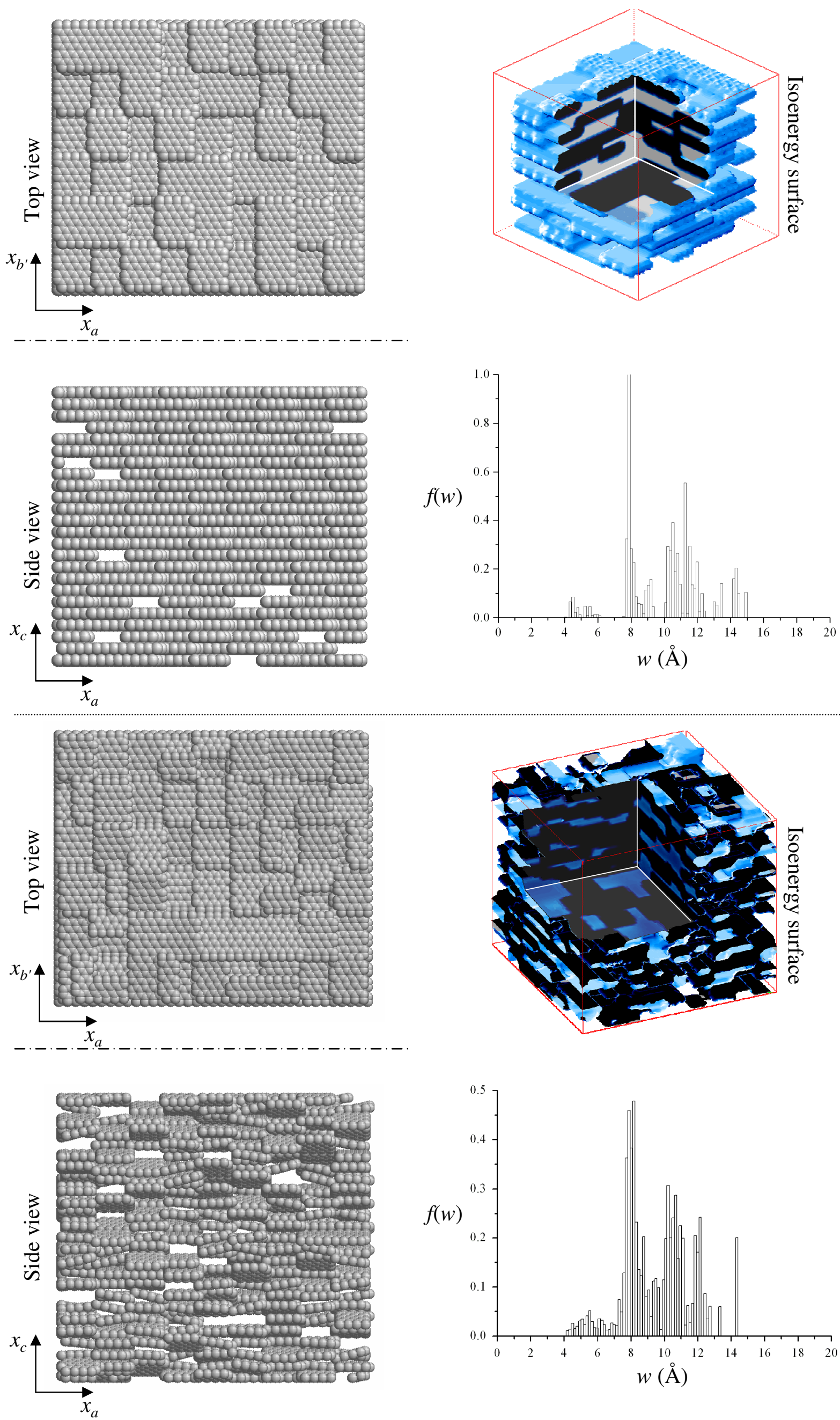

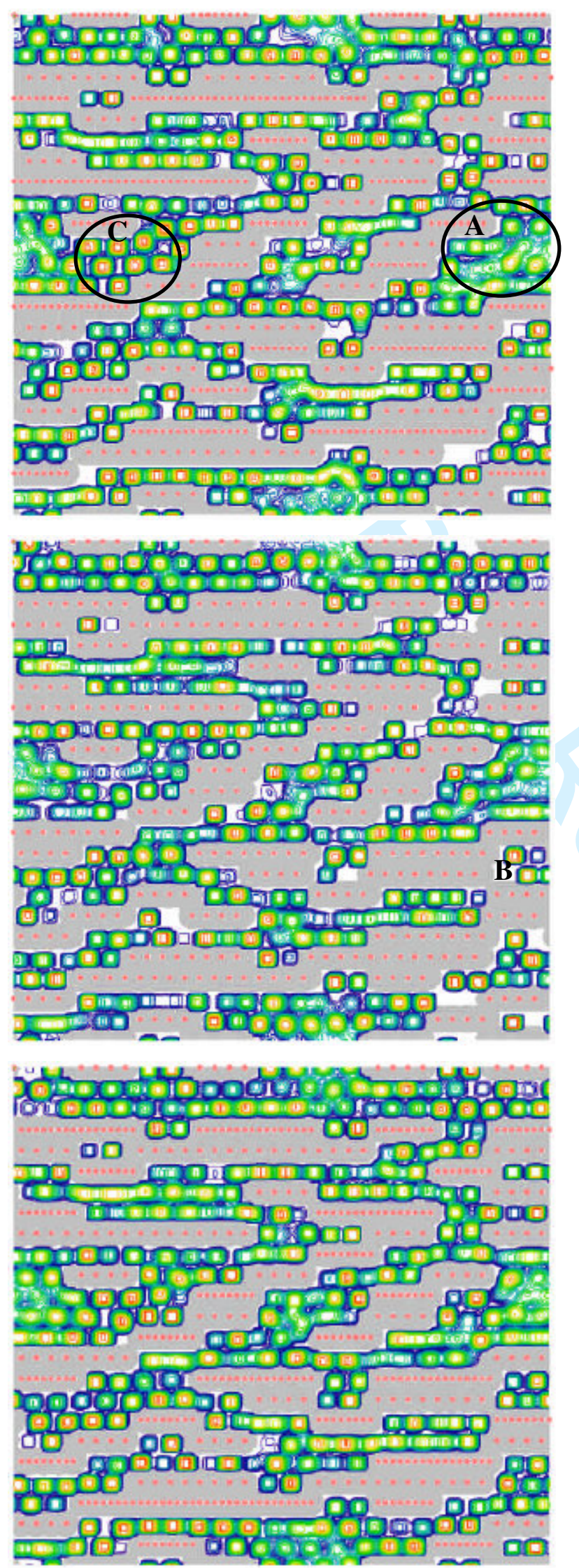

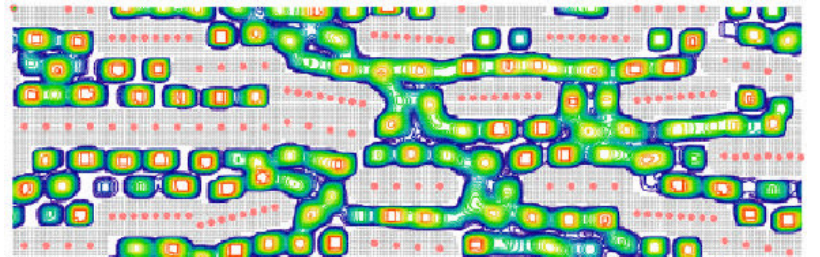

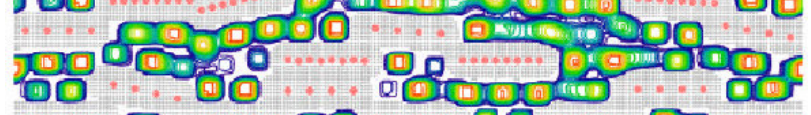
… boge 1900 1000 . og 000000 I00 -

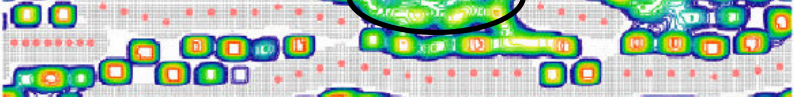
down son S30 acs og $00^{\circ}$ (6)

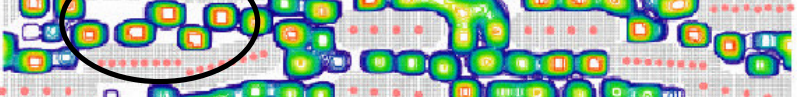

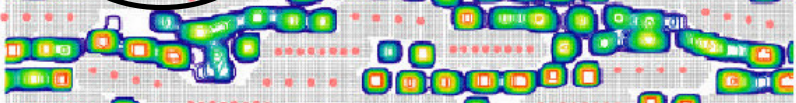

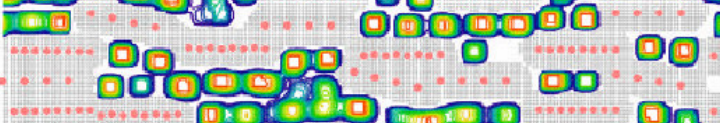

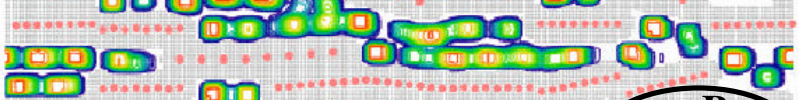

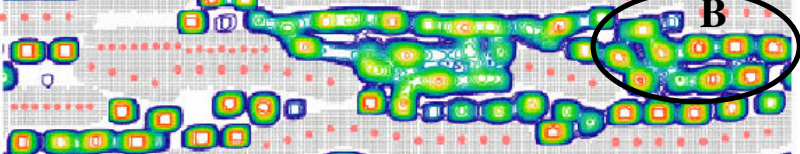

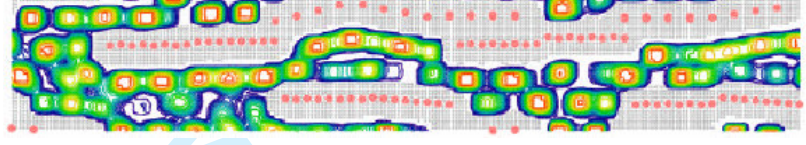

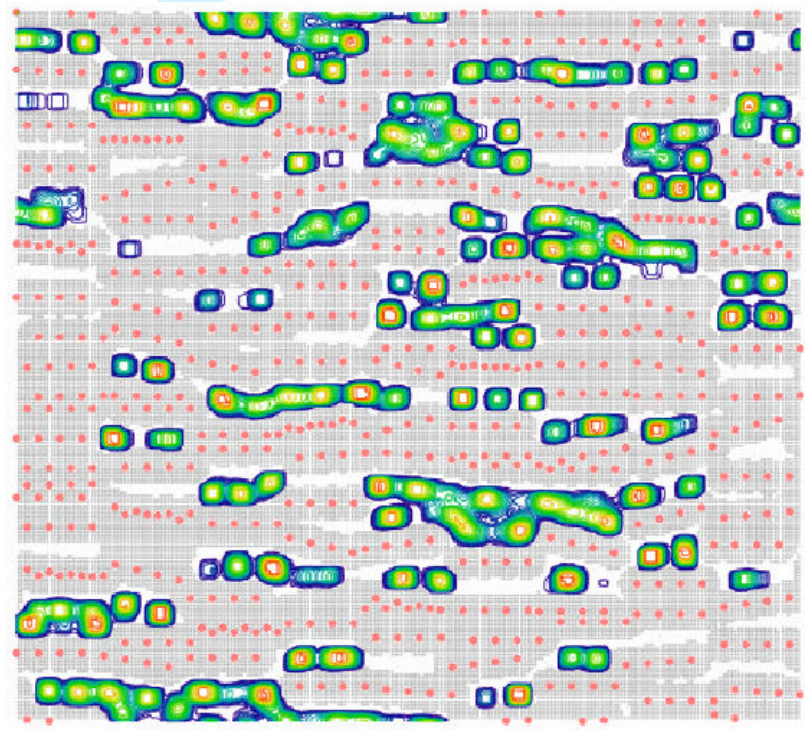
$\begin{array}{llllllllllll}5.0 & 3.5 & 2.3 & 1.6 & 1.0 & 0.7 & 0.5 & 0.3 & 0.2 & 0.1\end{array}$ 

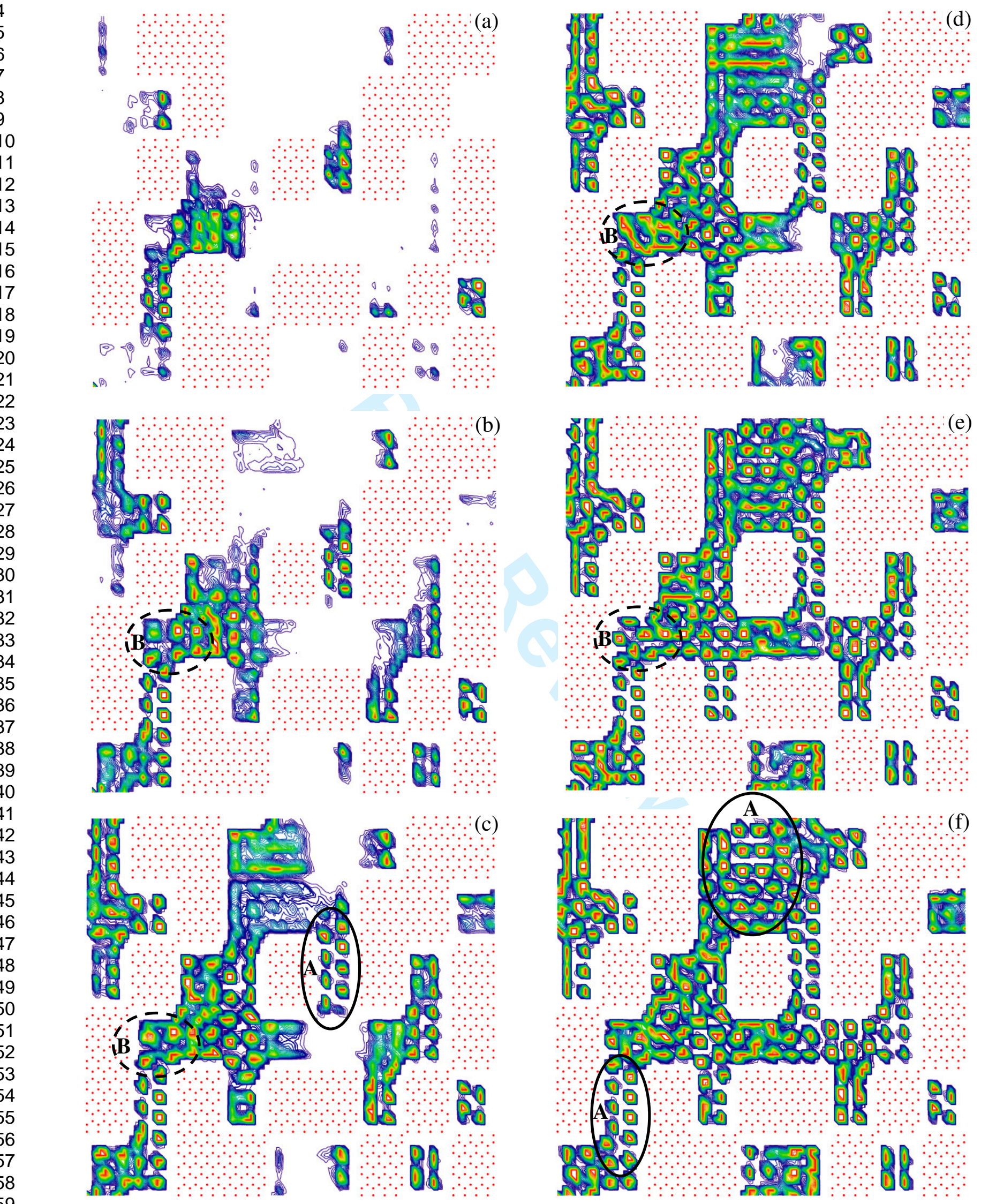

(e)
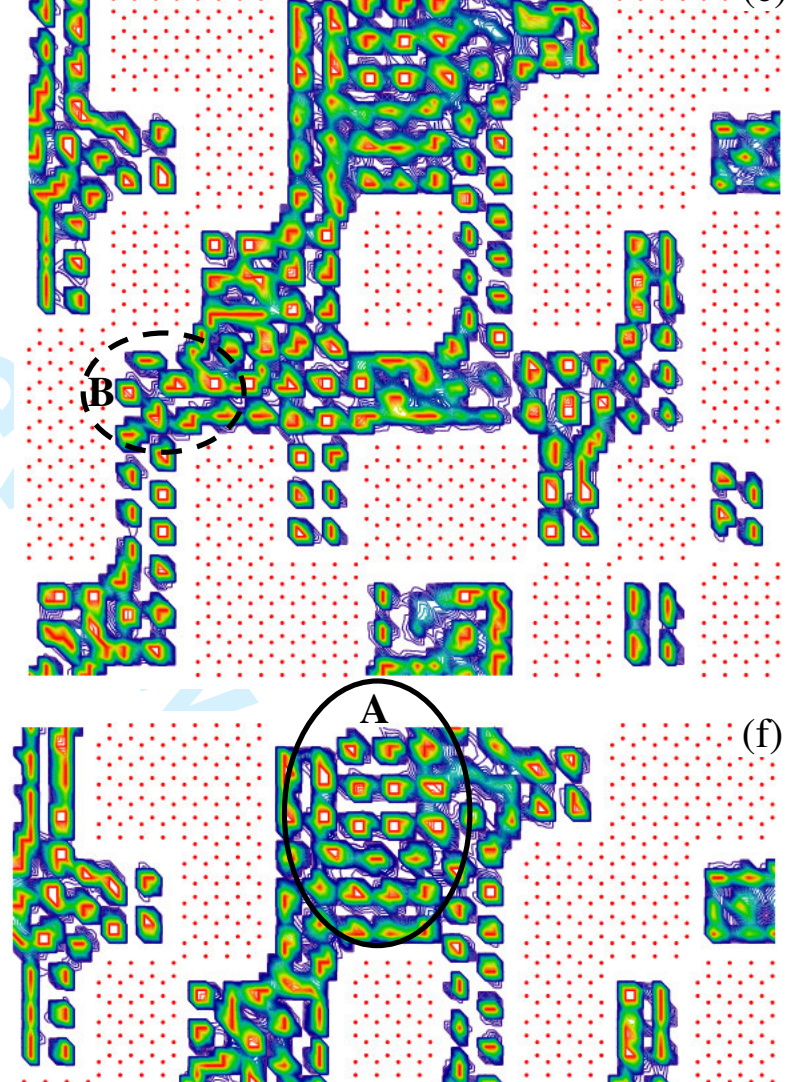

(f)

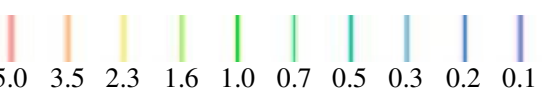




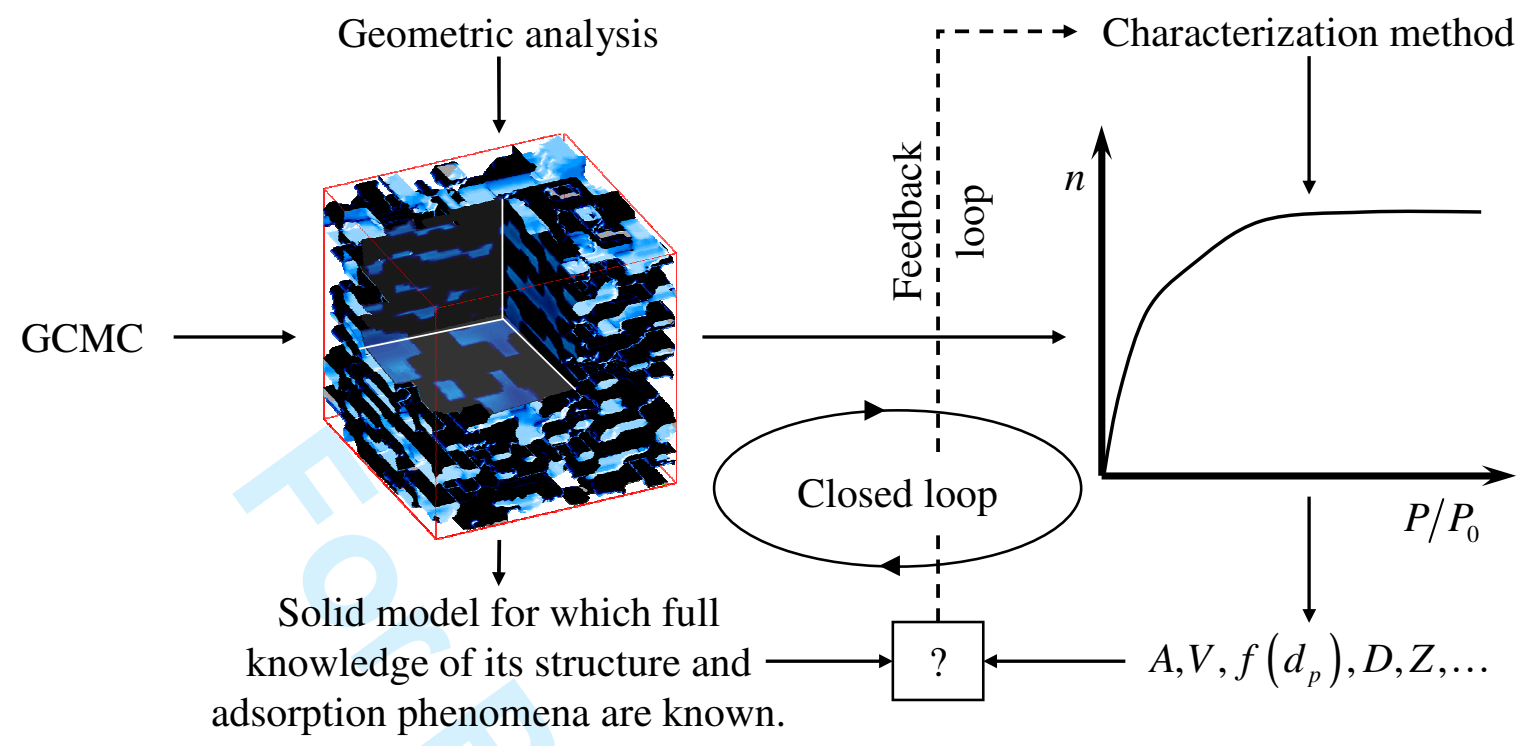




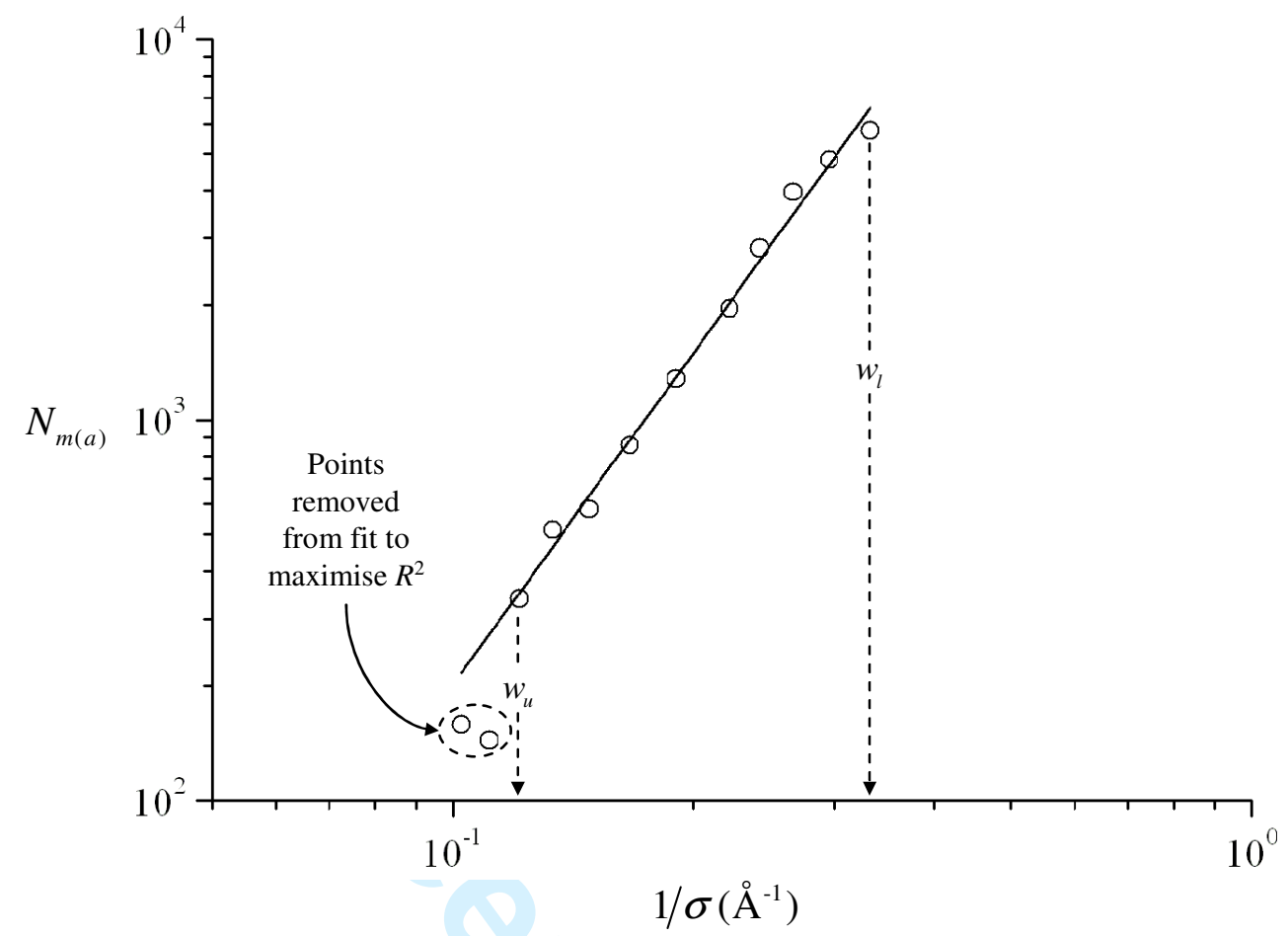




\section{Page 43 of 46}

1
2
3
4
5
6
7
8
9
10
11
12
13
14
15
16
17
18
19
20
21
22
23
24
25
26
27
28
29
30
31
32
33
34
35
36
37
38
39
40
41
42
43
44
45
46
47
48
49
50
51
52
53
54
55
56
57
58
59
60

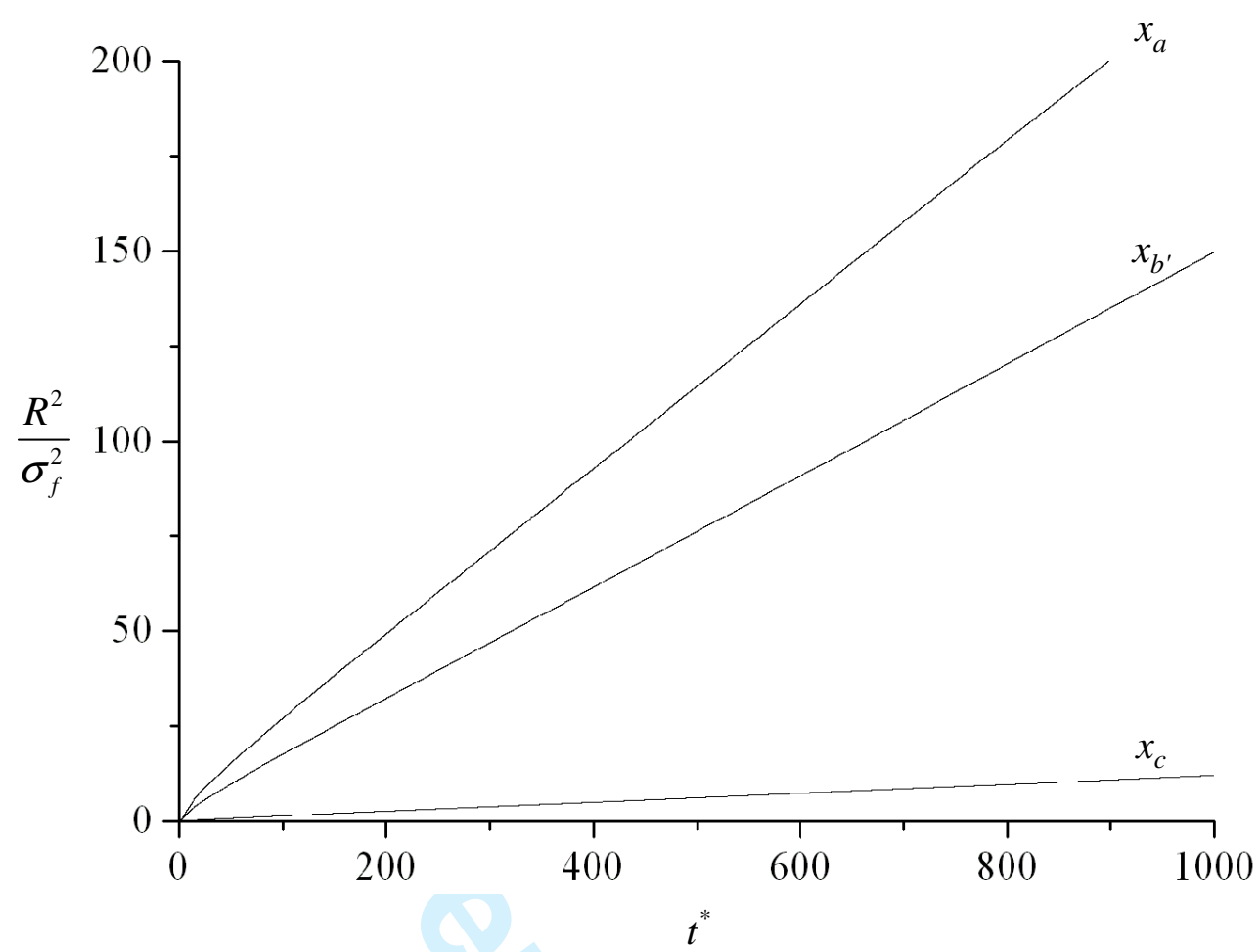



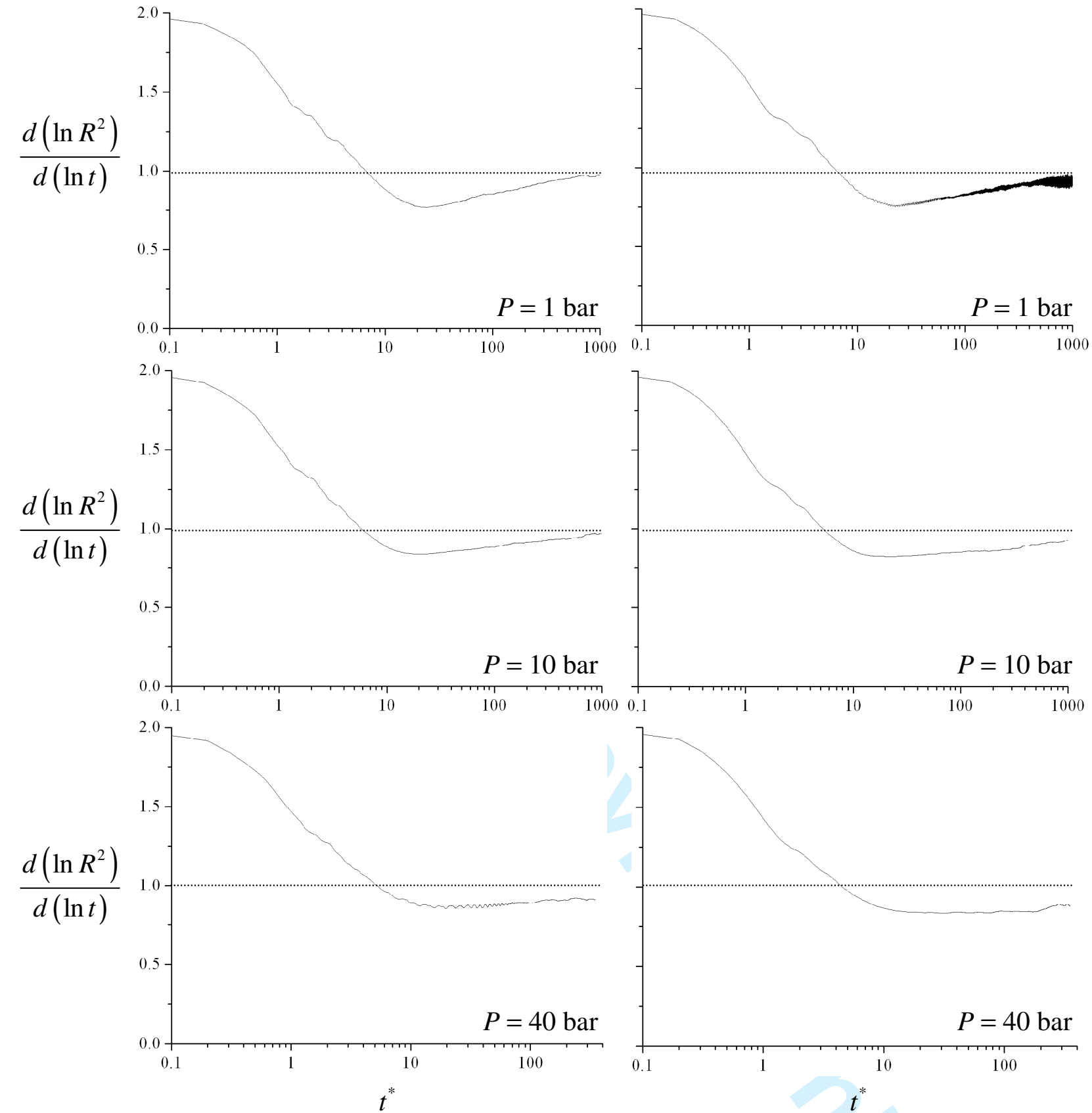


\section{Page 45 of 46}

1
2
3
4
5
6
7
8
9
10
11
12
13
14
15
16
17
18
19
20
21
22
23
24
25
26
27
28
29
30
31
32
33
34
35
36
37
38
39
40
41
42
43
44
45
46
47
48
49
50
51
52
53
54
55
56
57
58
59
60
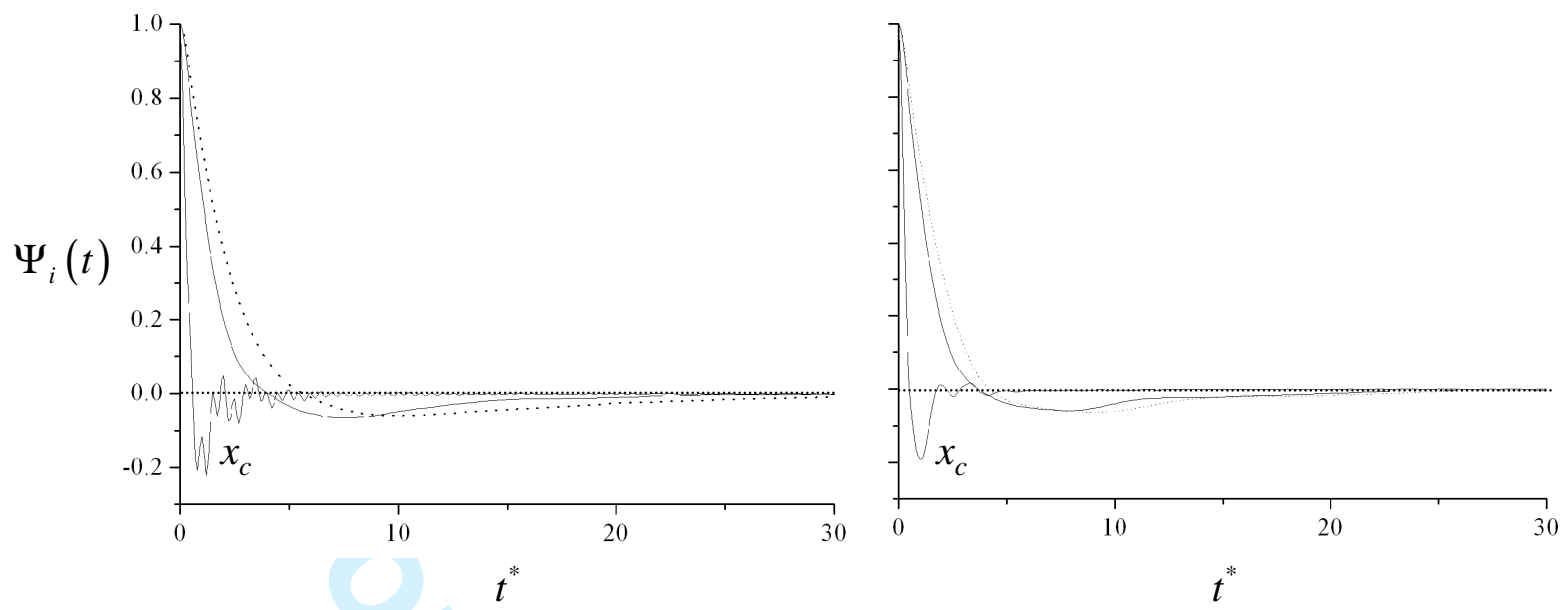

19

0

22

23

25

26

27

28

29

31

32

33

34

35

36

37

38

39

40

42

43

44

45

47

48

49

50

52

53

54

56

57

58 


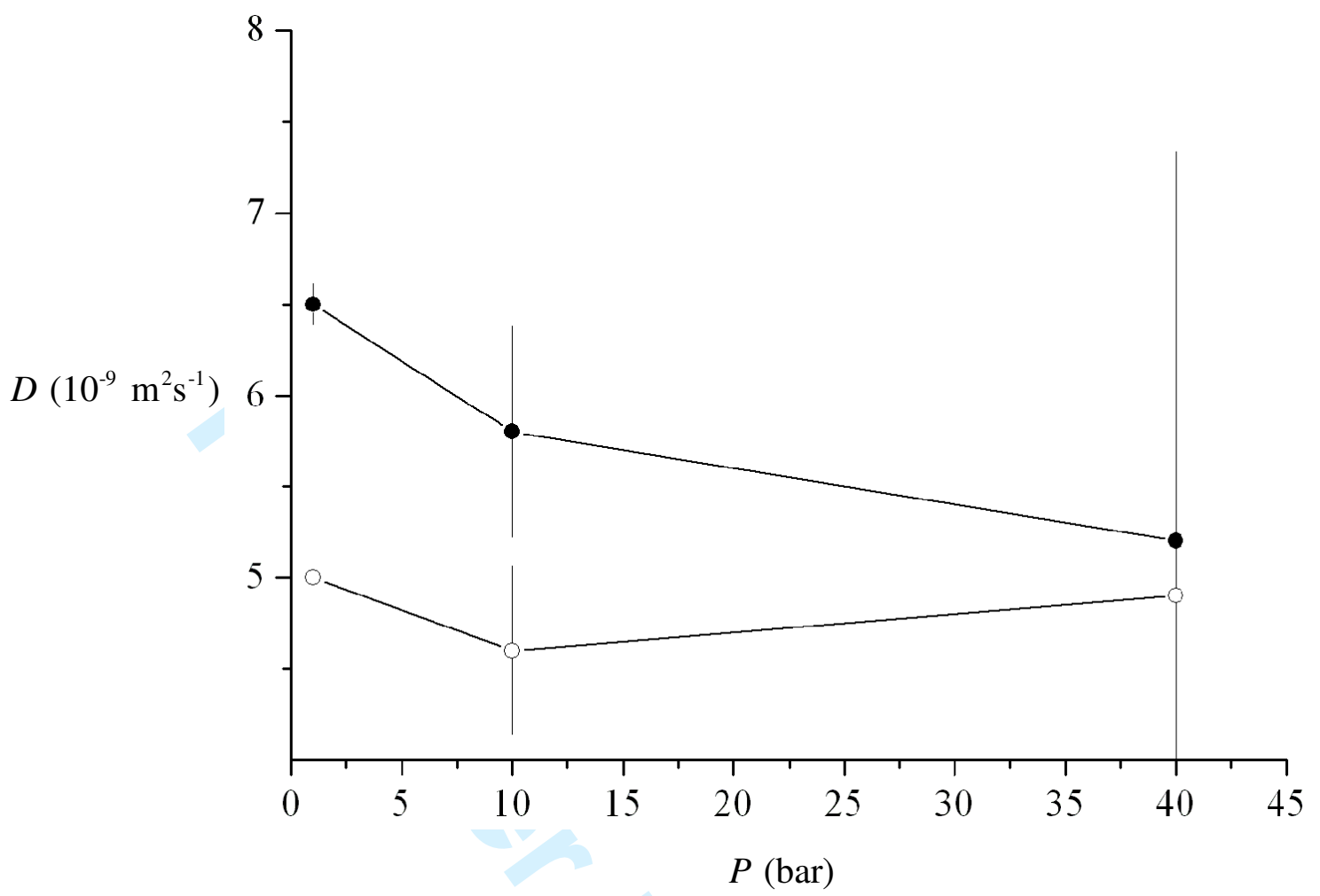

